\title{
Structural and optical characterisation of planar waveguides obtained via Sol-Gel
}

F. Rey-García, C. Gómez-Reino, M.T. Flores-Arias, G.F. De La Fuente, W. Assenmacher, W. Mader

\section{ABSTRACT}

Planar waveguides of $\mathrm{SiO} 2: \mathrm{TiO} 2$ (multilayer structure) and $\mathrm{SiO} 2: \mathrm{CeO} 2$ (thick layer) were prepared onto commercial glass substrates using a sol-gel technique combined with dip-coating. These glassy coatings were structural characterised by Transmission Electron Microscopy (TEM) Energy Dispersive X-ray analysis and by Confocal Microscopy. Thicknesses of $1230 \mathrm{~nm}$ and 4,15 $\mu \mathrm{m}$ and refractive indices of 1.59 and 1.48 for $\mathrm{SiO} 2: \mathrm{TiO} 2$ (70:30) and $\mathrm{SiO} 2: \mathrm{CeO} 2$ (95:5) waveguides were obtained, respectively, by Spectroscopic Ellypsometry. Losses of $0.8 \mathrm{~dB} / \mathrm{cm}$ were measured by double prism method in the $\mathrm{SiO} 2: \mathrm{CeO} 2$ system.

Keywords: Planar waveguides, sol-gel technology, transmission electron microscopy

\section{INTRODUCTION}

The preparation of planar waveguides has attracted considerable attention because of their possible use and applications in photonics devices in the field of communications and data transmission. Ion-exchange and chemical or physical vapor deposition methods have been extensively used to develop this kind of materials. Recently, laser deposition and direct writing techniques have been also employed to obtain planar waveguides on glass substrates of different compositions. These techniques have high economical cost and the compostions used raise the process complexity.

Recently researchers have focused their attention on glasses based on oxides of heavy metals ( $\mathrm{TeO} 2, \mathrm{GeO} 2$, $\mathrm{Bi} 2 \mathrm{O} 3, \mathrm{PbO}$, etc.) as promising materials for photonic devices. But the obtention of these materials suppose the obtaining of bulk materials, special reactives (heavy metals are restricted in use by European union) or the use, in the most of cases, of special atmospheres or high melting temperatures.

An alternative method to obtain planar waveguides is based on sol-gel technology. This technology is widely used in

different fields of life, from materials science to biological applications; there are developed bisosensors, bulk

materials, superconductors, fuel cells, ceramic composites etc; performing a wide range of

compositions. In particular, this technology permits us to obtain coatings with controlled thickness and refractive index.

The sol-gel process is suitable to produce both thin and thick films by single and multideposition techniques. A several number of papers dealing with planar waveguides fabricated with this method have been reported in literature.

The microstructure of sol-gel derived coatings, thus their optical properties, is affected by different parameters involved in their synthesis. These include starting materials, and thermal processing conditions, among others. The optimization and control of all these parameters should allow us the production of efficient sol-gel derived planar waveguides. Following this concept, one important advantage provided for the sol-gel technology is the lower surface roughness obtained for the coatings. This fact allows us to drastically reduce guiding losses for geometry. The roughness is investigated by confocal microscopy.

Only few papers have employed the transmission electron microscopy to study the structural characteristics between solgel films and substrates and the process involved in the union between them; the most of the literature reports the study of thin films, their interfaces and the crystalline structures presents. 
The aim of this work is to prepare planar waveguides by sol-gel technology. The sol-gel film structural characteristics; the interface structure and the processes involved in the union between materials are studied by electron microscopy and optical confocal microscopy.

\section{EXPERIMENTAL}

Hybrid silica-titania (STi sol) and methacrylate-silica-cerium oxide (SCe sol) sols were prepared by acid catalysis following different routes. Low-hybrid sol silica-titania incorporates methyl-groups as the organic part responsible for increasing the critical thickness of the coatings. On the other hand, highly organic hybrid sols (SCe sol) are obtained from a hybrid alkoxide, 3-methacryloxypropyl trimethoxysilane (MPS), where methacrylate groups can be partially polymerised in the liquid in order to develop two interpenetrating and bonded organic and inorganic networks.

STi sol was prepared by complexing tetraisopropoxytitanate (TISP) with acetic acid and adding methyltriethoxysilane (MTES) in a proportion of 70:30 and acidified water in a dropwise manner, to complete synthesis reactions.

Highly organic hybrid sol (SCe sol) was prepared following the process described in. The methacrylate groups are partially polimerised in liquid using Irgacure-184 as initiator.

Coatings were deposited on $5 \mathrm{~mm}$ thick commercial window glass by dip-coating varying the withdrawal rate between 12.5 and $25 \mathrm{~cm} / \mathrm{min}$. Firstly, the substrates were cleaned in an ultrasonic bath with absolute ethanol for $15 \mathrm{~min}$. The silica-ceria film was cured at $150^{\circ} \mathrm{C}$ during 1 hour. Multilayer coatings were prepared for the silica-titania composition.

Substrates were dip-coated with five-layers of the SiTi sol and a constant withdrawal rate of $25 \mathrm{~cm} / \mathrm{min}$. Intermediate heat treatments at $450^{\circ} \mathrm{C}$ during 15 min were applied between coatings, followed by a final treatment at $450^{\circ} \mathrm{C}$ during 1 hour.

Thickness (e) and refractive index (n) were respectively measured by profilometry (Talystep, Taylor-Hobson, UK) using a variable angle spectroscopic ellipsometer (Woollam M2000U).

Values measured are shown in Table 1.

Table 1: Refractive index $(\lambda=633 \mathrm{~nm})$ and thickness for coatings studied.

\section{System Molar composition Refractive index (n) Thickness (nm)}

STi monolayer 70:30 1.63 358

STi multilayer 70:30 1.59 1230

SCe 95:5 1.47 4150

Microstructural studies were performed with a FEG/UT-STEM Phillips CM300, field emission gun and ultra twin lens and scanning unit operated at $300 \mathrm{kV}$ and equipped with an energy dispersive $\mathrm{x}$-ray detector (HPGe detector, Noran Inc.) and analytical system (energy dispersive X-ray unit, Noran Inc.).

Thick layer SCe with $4.15 \mu \mathrm{m}$ in thickness and refractive index value of 1.47 was selected to study optical waveguide efficiency. These measurements were performed using the double prism method reported in (Fig. $1)$. 


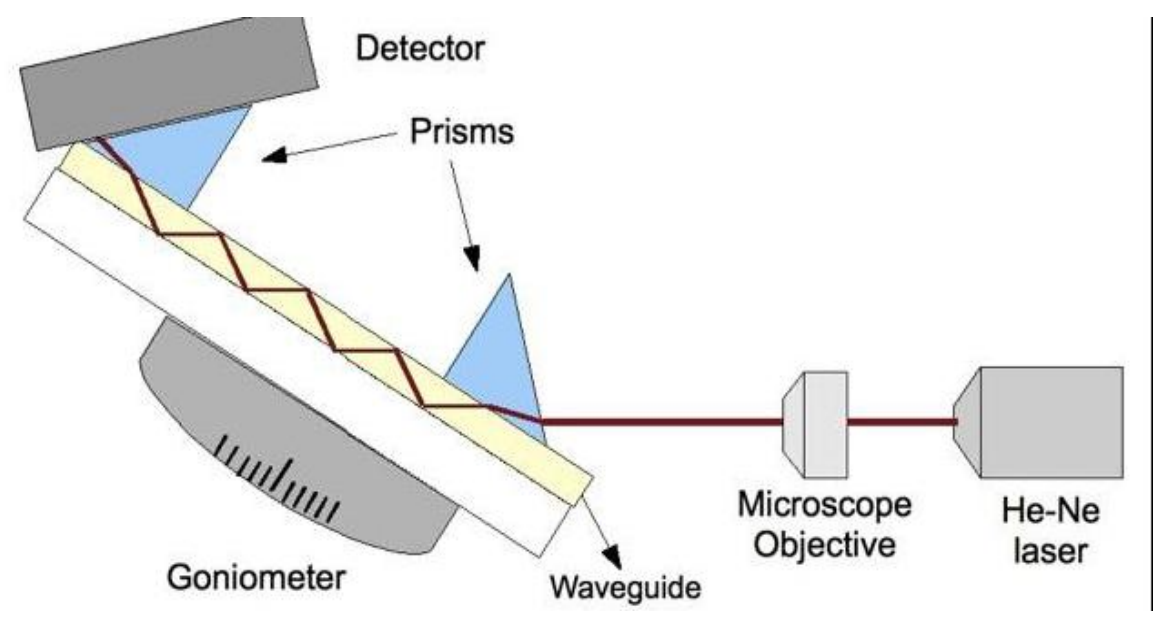

Figure 1: Schematic drawing of the experimental double-prism set-up use to determine the optical efficiency of the

Sol-Gel waveguides obtained in this work. Elements are identified in the drawing.

\section{RESULTS AND DISCUSSION}

We have prepared three samples to evaluate the quality of planar waveguides obtained via Sol-Gel. The first sample

studied was a 70:30 SiO2:TiO2 multilayer, noted by STi-MC; moreover, we have previously characterised a monolayer sample with the same composition (STi) to study the process involved in the union of materials. The last sample studied was the $\mathrm{SiO} 2-\mathrm{Ce} 2 \mathrm{O} 3$ thick layer, name as $\mathrm{SCe}$.

The study of the sample STi allows us to observe a good adherence between sol coating and commercial glass substrate (Fig. 2). We can observe a geometrically defined layer; it will result in lower losses of guided light due to geometry. Moreover, a darker zone in the coating is observed by increasing the magnification of the microscope; we can study in detail this event assisted by EDX analysis (Fig. 3). A process of diffusion of alkali cations is observed; $\mathrm{Na}$ ions move to the coating from the glass. Moreover, the Ti proportion in the area close to interface is higher than the external area of the coating, opposite to the Si distribution. The second factor which promotes the darker colour is the amount of alkali cations at this area, just a little higher than the rest of the coating. Consequently, the diffusion of alkali cations promotes a phase rich in silicon respect to the rest of the substrate. Then, this diffusive process is responsible of the chemical union between materials.

We develop the multilayer structures in order to obtain a thickness adequately to achieve the entering of light and the subsequent guiding of the beam through the sol-gel layer. The study of the silica-titania multilayer structure shows the possibility to obtain a homogeneous thick layer from the stacking of successive thin layers. 


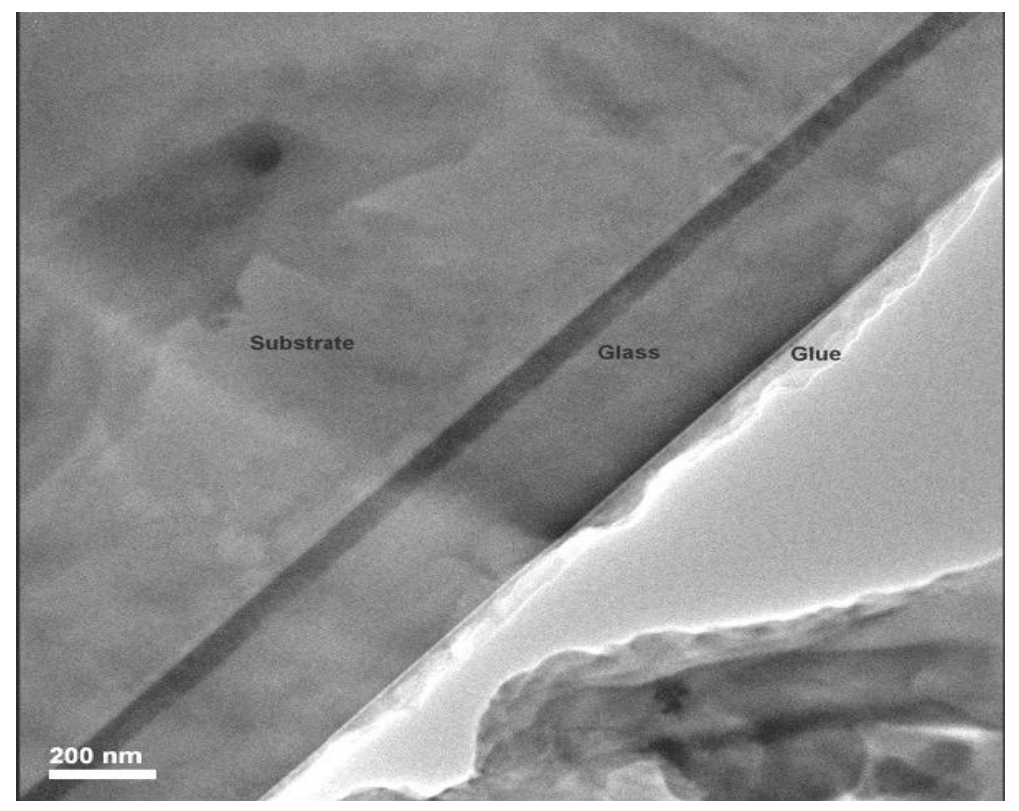

Figure 2: TEM micrography of the STi sample. Interface is well-defined; we can observe a darker area in the film due to a higher amount in Ti and alkali cations.

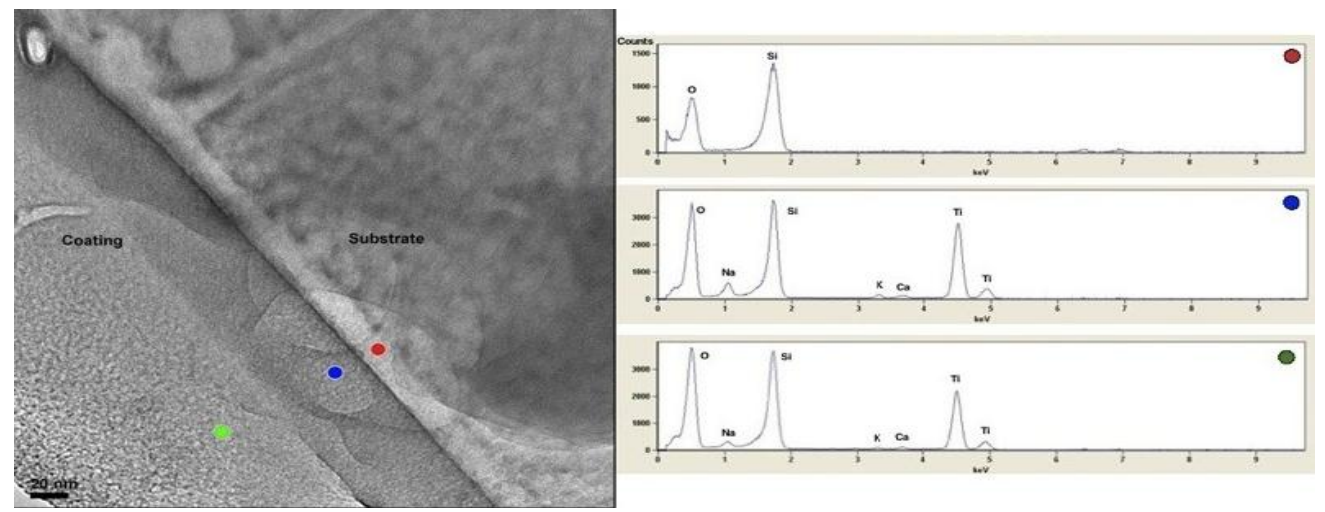

Figure 3: TEM micrography at the interface of the STi sample and the corresponding spectra of the marked places.

The study of the interface between layers allows us to check a homogeneous and continuous union between layers (Fig. 4). The texture of the layer is similar between them and the difference in colour is due to a different thickness of the TEM specimen. Thus, this effect leads to confirm the possibility to obtain one thick layer from the stacking of several thin layers.

The study of the highly hybrid coating produces the same conclusions. We made the EDX analysis at the interface to

probe if the diffusion process was common at the sol systems. In the study of the interface between materials for SCe sample we have observed again the diffusive process of alkali cations and the Si-rich zone at the upper substrate area (Fig. 5). This coating is more bright respect to the silica-titania due to its higher amount in organic compounds. Some particles of potassium chloride come from contamination at the TEM preparation step are presents. 


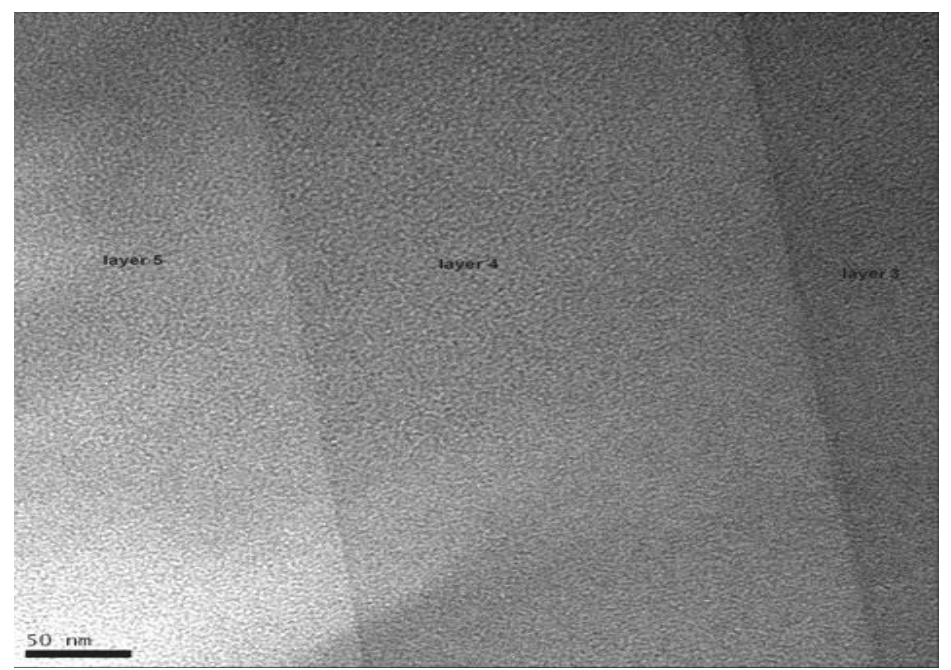

Figure 4: TEM micrography of layers 3 to 5 in the sample STi-MC. We can observe concrete interfaces. The layers

seem homogeneous between they like a continuous material.

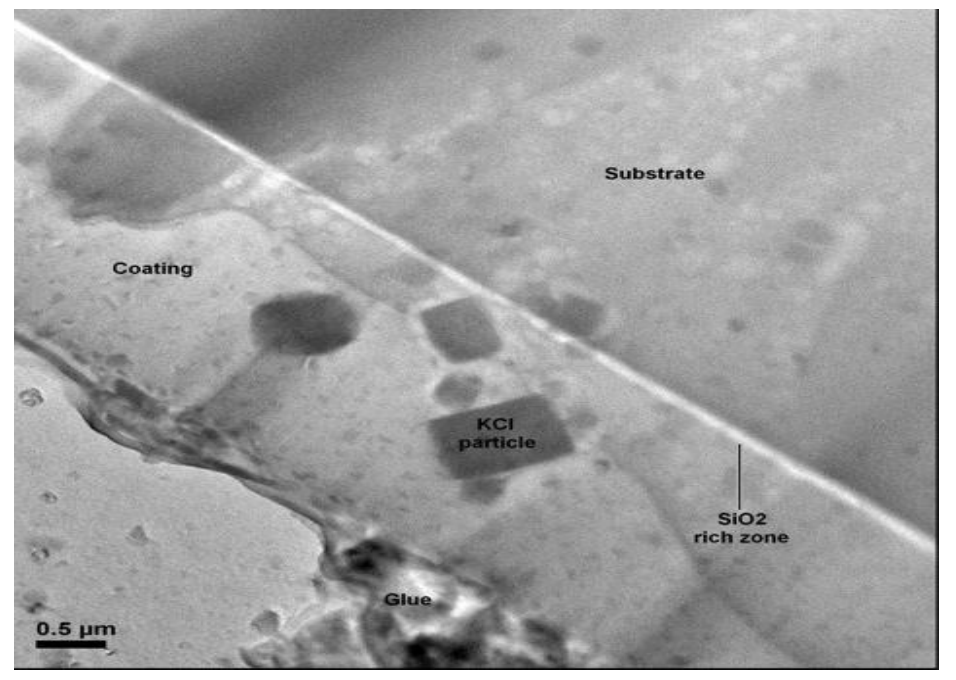

Figure 5: TEM micrography of the sample SCe. The different phases present are denoted.

No crystalline phases were found at the specimens studied. The presence of crystalline phases at the films will provide the diffraction of light, and, therefore it negatively affects the efficiency.

In Figure 6 , we can see the light from a He-Ne laser $(\lambda=633 \mathrm{~nm})$ at the silica-ceria thick layer; we can observe the light concentrated at the exit. This coating was employed to waveguide efficiency measurements applying the double prism method [50-54]. The beam of a He-Ne laser emitting at $633 \mathrm{~nm}$ with a nominal power of $35 \mathrm{~mW}$ was introduced into an entry prism by focusing it through a microscope objective (20x, NA $0.4)$. The entrance angle into the prism $(n=1.779$, NSFL-11, Edmund Optics) was defined mathematically using the Snell's law, resulting in a value of $1^{\circ} 42^{\prime}$ with respect to the normal to the prism hypotenuse. A second exit prism was placed $2 \mathrm{~cm}$ away. Coupling between the prisms and the waveguide was achieved with an index adjustment liquid $(\mathrm{n}=1.56)$. 


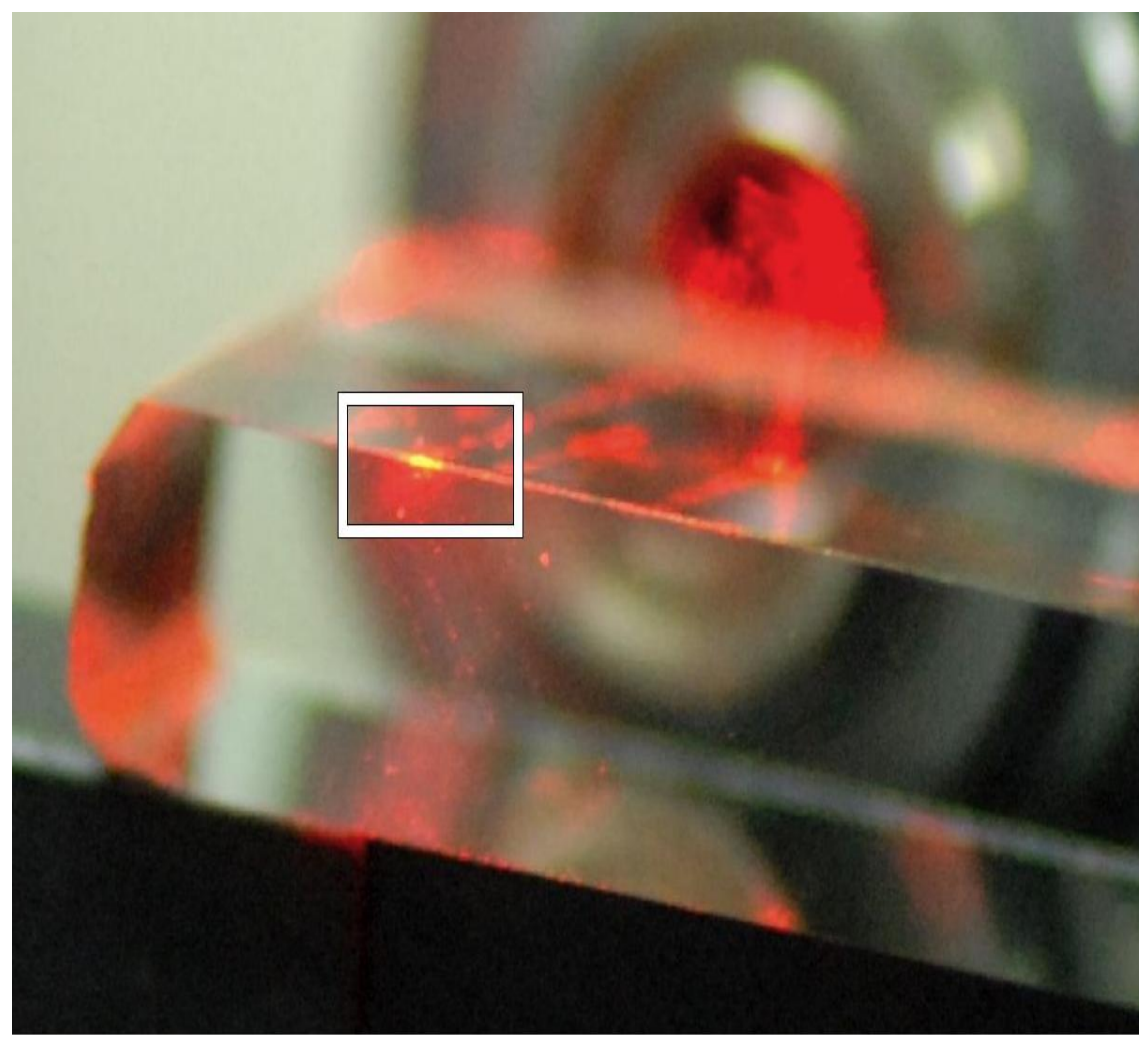

Figure 6: Image of the SCe sample guiding the light of a He-Ne laser beam, coupled via microscope objective. We can observe an exit point with high concentrion of light.

A laser powermeter (Beam profiler, Thorlabs $\mathrm{GmbH}$ ) was used to measure the entrance and exit laser beam power. The entrance power $(24 \mathrm{~mW})$ was determined taking into account losses due to the absorption of glass substrate. Power exit of $17 \mathrm{~mW}$ was achieved at the output of the second prism coupled to the waveguide. This measure results in an efficiency value for the waveguide of $0.8 \mathrm{~dB} / \mathrm{cm}$. This value is approximate, since coupling losses at the entry prism were not evaluated, and it was assumed that $100 \%$ of the laser power was introduced into the prism.

\section{CONCLUSIONS}

Planar waveguides of $\mathrm{SiO} 2: \mathrm{TiO} 2$ and $\mathrm{SiO} 2: \mathrm{CeO} 2$ were prepared by sol-gel technology. The different composition layers were deposited over commercial window glass by dip-coating varying the withdrawal rate. In particular, we present $\mathrm{SiO} 2: \mathrm{TiO} 2$ mono-layer and multi-layer and $\mathrm{SiO} 2: \mathrm{CeO} 2$ monolayer with refractive index values of 1.63, 1.59 and 1.47 for a wavelength of $633 \mathrm{~nm}$, and thickness of $352 \mathrm{~nm}, 1230 \mathrm{~nm}$ and $4150 \mathrm{~nm}$, respectively. A microstructural study were performed by TEM obtaining that, in all cases, the interfase between layers are homogeneous and continuous. No crystalline phases were found, that will became in a better guidance of light. In order to test viability of the layers fabricated as waveguides, efficiency measurement were done with the double prism method for the $\mathrm{SiO} 2: \mathrm{CeO} 2$ layer, obtaining a value of $0.8 \mathrm{~dB} / \mathrm{cm}$.

\section{ACKNOWLEDGEMENTS}

The authors acknowledge funding from MICINN (TEC2006-10469, CEN 2007-2014, SURFALUX SOL00030930 and MAT2010-18519), from DGA (Group of Excellence in Laser Material Processing and Characterisation) and XUNTA DE GALICIA (INCITE08PXIB206013PR). 
